\title{
Using lock-in thermography to investigate stimuli-responsive nanoparticles in complex environments
}

Mathias Bonmarin ${ }^{\mathrm{a}, \mathrm{b}}$, Lukas Steinmetz ${ }^{\mathrm{c}}$, Fabrizio Spano ${ }^{\mathrm{a}}$, Christoph Geers ${ }^{\mathrm{b}, \mathrm{c}}$

${ }^{a}$ Zurich University of Applied Sciences, School of Engineering, Technikumstrasse 5, 8400 Winterthur Switzerland.

${ }^{b}$ NanoLockin GmbH, Adolphe Merkle Institute, Passage du Cardinal 1, 1700 Fribourg Switzerland

${ }^{c}$ University of Fribourg, Adolphe Merkle Institute, Chemin des Verdiers 4, 1700 Fribourg Switzerland

*mathias.bonmarin@zhaw.ch

The use of nanoparticles (NP) has been dramatically rising in the recent years and NPs can nowadays be found in various products ranging from food to composite materials or cosmetics $[1,2]$. Currently, the most frequently employed NP types are titania $\left(\mathrm{TiO}_{2}\right)$, as a typical color additive, silica $\left(\mathrm{SiO}_{2}\right)$, as anticoagulation agent, and silver $(\mathrm{Ag}) \mathrm{NPs}$, which are added to textiles, due to their antimicrobial properties. Because of their outstanding physical and mechanical properties carbon-containing nanomaterials, such as graphene and carbon nanotubes, have also experienced a surge in industrially relevant applications. About $30 \%$ of the NPs are suspended in liquids, ranging from water to creams and lotions to car lubricants, followed by applications containing surface-bound (e.g., in textiles) NPs and finally nanocomposites (e.g., polymer-CNT composites). Together with biological and physiological fluids, these matrices render the detection and quantification of NPs fairly complex [3]. The broad range of chemical and biological compositions of these environments, including $\mathrm{pH}$ and ionic strength are often detrimental to the colloidal stability of NPs, potentially causing aggregation or even dissolution effects and therefore render NP analysis fairly challenging. Man-made, so-called 'engineered' NPs can be precisely tailored with respect to specific and desired properties. These materials are often stimuli-responsive meaning that they demonstrate the ability to generate heat upon a stimulus: alternating magnetic fields (AMF) for magnetic particles like superparamagnetic iron oxide NPs (SPIONs), and light in the UV, visible or NIR range for plasmonic NPs like gold spheres and other photothermal particles. Such a behavior 
makes them excellent candidates for active thermal imaging, an investigation method that is using an infrared camera to record the transient thermal emission of a sample surface.

In this work we present lock-in thermal imaging (LIT) [4] an averaging active thermal imaging method allowing to reach high sensitivity, as a promising investigation tool for NPs embedded in complex medium. We demonstrate the LIT setups developed in our laboratory, the algorithms employed and discuss potential schemes to compensate for non-steady initial heating, emissivity variations or high absorptive environments. Finally, we compare LIT with other methods currently employed to investigate NPs in complex environments.

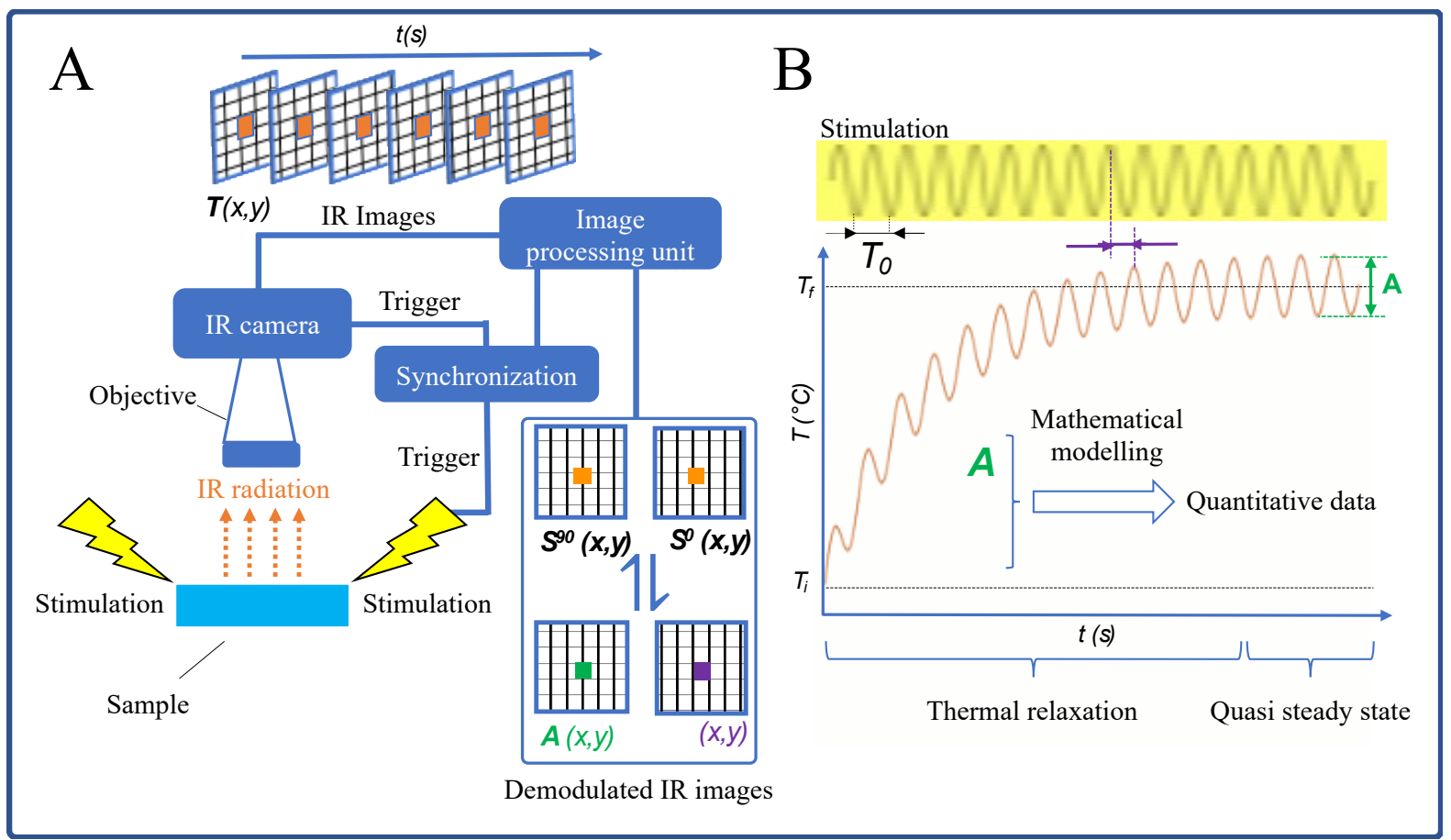

Figure 1: The lock-in thermography principle (LIT). A: Schematic description of a typical LIT setup. The thermal radiation emitted by the surface of the stimulated sample is captured by the infrared camera objective and converted into temperature maps or thermogramms $T(x, y)$. The resulting images are processed to calculate an amplitude $A(x, y)$ in Celsius or Kelvin and a phase image $\phi(x, y)$ in Radian from in-quadrature $S^{90}(x, y)$ and in-phase images $S^{0}(x, y)$. For best performances, camera acquisition and thermal stimulation are synchronized by a trigger signal. B: Transient temperature signal of a single pixel of the infrared images. The temperature oscillates at the same frequency as the stimulation signal but exhibits a particular amplitude $A$ and phase difference $\phi$. At the beginning of the stimulation, the signal experiences a non-steady-state phase called thermal relaxation where it starts at temperature $T_{i}$ and steadily increases before oscillating around a mean value $T_{f}$ in a quasi-steady-state phase. Using mathematical modelling, the amplitude and/or phase information can be converted to valuable quantitative data. 


\section{Active thermal imaging and Lock-in thermal imaging (LIT)}

In active or dynamic thermal imaging, the sample is monitored in the transient state following a thermal stimulation [5]. Active thermal imaging is a well-known technique in engineering, widely used for the non-destructive testing of materials (NDT). The source employed to generate the thermal stimulation depends on the sample under investigation. To localize water ingress for example, microwaves are particularly suited $[6,7]$, whereas eddy currents will be more appropriate to detect cracks in metallic materials [8]. We demonstrated that active thermal imaging is a promising tool for engineered NPs [9-14]. Lock-in thermal imaging (LIT) records the sample surface temperature during the application of a harmonic heating stimulus at a defined frequency [15]. Figure 1 describes the principle of a lock-in thermography setup. The sample is stimulated at a frequency $f_{0}=1 / T_{0}$ called "stimulation frequency" while the thermal radiation from the sample surface is captured by an infrared camera (see Part A of Figure 1). The recorded infrared images are first converted into temperature maps or thermograms $T(x, y)$ before being processed according to the digital lock-in principle resulting in an in-quadrature $S^{90}(\mathrm{x}, \mathrm{y})$ and an in-phase image $S^{0}(\mathrm{x}, \mathrm{y})$ that can be converted into a phase image $\phi(x, y)$ and an amplitude image $A(x, y)$.

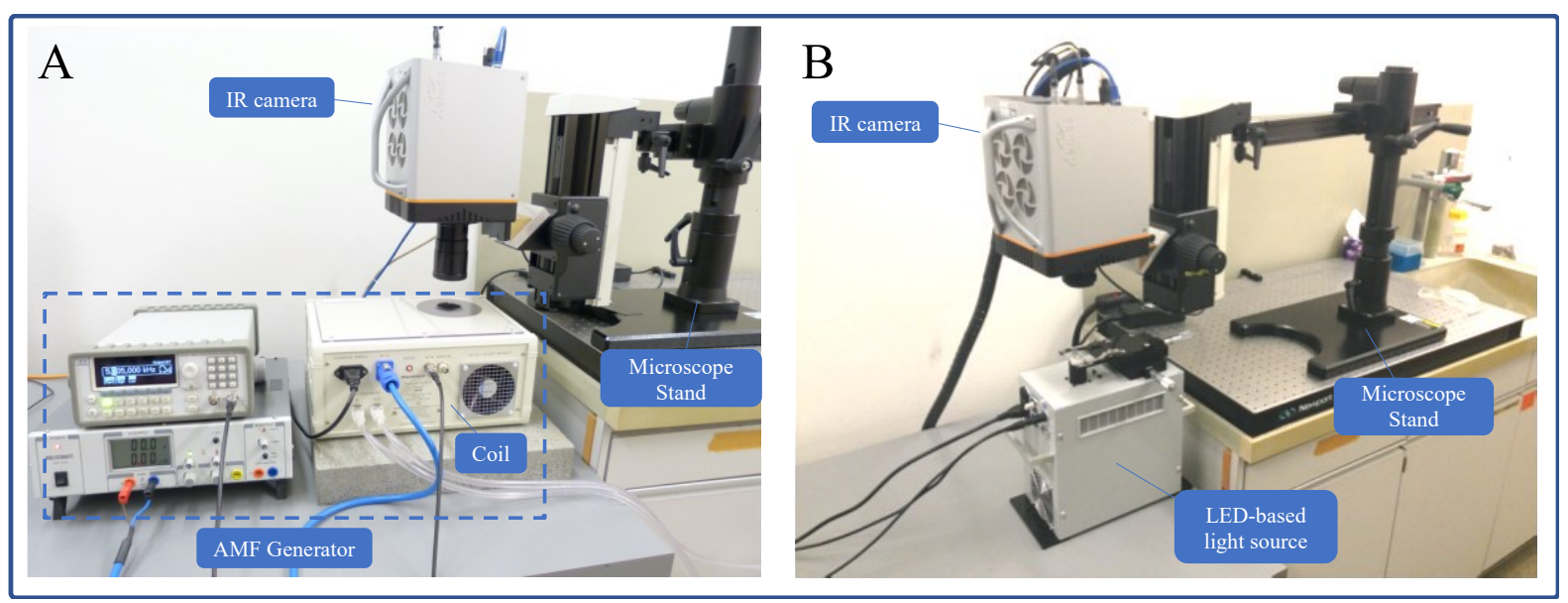

Figure 2: A: Picture of the lock-in thermal imaging apparatus developed to investigate magnetic NPs, such as SPIONs. B: Picture of the lock-in thermal imaging apparatus developed to investigate plasmonic and other photo-thermal NPs.

The phase is a map of the phase angles between the stimulation signal and the harmonic temperature response of the sample surface, whereas the amplitude gives the oscillation amplitude (see Part B of Figure 1).

Various lock-in thermal imaging instruments have been developed depending on the application and sample being investigated. Figure 2 presents a picture of the two laboratory setups specifically developed to investigate stimuli-responsive NPs. Magnetic NPs are 
stimulated using an AMF. We use a commercial generator (MagnethermTM V1.5, nanoTherics) that comprises a function generator (SFG-2004, GW Instek), a water-cooled coil and a laboratory power supply (EA-PS 3032-20B, EA Elektro Automatic) (Part A of Figure 2). For photothermal NPs, we designed and built a custom LED-based light source (see Part B of Figure 2 and the paragraph below dedicated to non-homogeneous stimulation). Infrared radiation of the sample surface is captured by an infrared camera (Onca-MWIR-InSb-320, XenICs). The Onca camera is based on an InSb array $(320 \times 256$ pixels $)$ and records infrared radiation between 3 and $5 \mu \mathrm{m}$ with a full frame rate $f_{\text {cam }}$ of up to $250 \mathrm{~Hz}$. The nominal thermal resolution of the camera is $0.017^{\circ} \mathrm{C}$. For this study we used the standard $25 \mathrm{~mm}$ focal length lens provided by the camera manufacturer. A frame grabber card (PCIe-1427, National Instruments) is used to transfer the infrared images in real time to a computer and to synchronize the infrared camera and the stimulation source respectively (Part A of Figure 1). The personal computer sets the stimulation frequency via the frame grabber card, while both the infrared camera and the stimulation source are working in slave mode. The setups depicted in Figure 2 use a quantum detector-based infrared camera. Quantum detectors transform absorbed photon energy directly into released electrons. Most commercial cameras are operating in the photovoltaic mode where photo-excited charge carriers are collected by a diode junction. Thermal detectors convert the far-IR radiation into heat causing electrically measurable changes in the electrical resistance of a bolometer. They are called microbolometers.

Quantum-based sensors demonstrate better performances than microbolometers in terms of sensitivity and speed, but their costs are prohibitive and microbolometers are therefore widely used in industry. As they are functioning in the rolling frame mode (meaning the image acquisition cannot be triggered) they have to act as the master clock synchronizing the stimulation source.

\section{LIT algorithm}

Many algorithms can be found in the literature to compute the phase and amplitude image. In synchronous narrow two-channels correlation, two factors are used to approximate the sine and cosine function

$$
S^{0^{\circ}}(x, y)=\frac{1}{n N} \sum_{i=1}^{N} \sum_{j=1}^{n} 2 \sin \left(\frac{2 \pi(j-1)}{n}\right) T_{i, j}(x, y)
$$




$$
S^{90^{\circ}}(x, y)=\frac{1}{n N} \sum_{i=1}^{N} \sum_{j=1}^{n} 2 \cos \left(\frac{2 \pi(j-1)}{n}\right) T_{i, j}(x, y)
$$

where $T_{i, j}(x, y)$ are the time-dependent infrared images, $n$ is the number of measurements per stimulation cycle, and $N$ is the number of stimulation cycles. $S^{0^{\circ}}(x, y)$ and $S^{90^{\circ}}(x, y)$ are the "in-phase" and "in-quadrature" images equivalent to the real and imaginary image resulting from a discrete Fourier transform. From $S^{0^{\circ}}(x, y)$ and $S^{90^{\circ}}(x, y)$ the amplitude $A(x, y)$ and phase image $\phi(x, y)$ can be calculated[15]:

$$
\begin{gathered}
A(x, y)=\frac{2}{N} \sqrt{S^{0^{\circ}}(x, y)^{2}+S^{90^{\circ}}(x, y)^{2}} \\
\phi(x, y)=\tan ^{-1}\left(\frac{S^{90^{\circ}}(x, y)}{S^{0^{\circ}}(x, y)}\right)
\end{gathered}
$$

When the stimulation source is synchronized with the infrared camera, and if the pulsation $\omega_{0}$ is low compared to the frame rate $(n \geq 10)$, synchronous narrow two-channels correlation is the optimal digital demodulation algorithm in terms of accuracy and computing time. This method demonstrates the advantage to be performed in real time and does therefore not require any storage of the infrared images. The first image $T_{0,0}(x, y)$ is usually subtracted before performing the demodulation.

To perform the synchronous narrow two-channels correlation, we implemented a custom software (LabVIEW, National Instruments) using the Vision Acquisition Software (VAS, National Instruments). The demodulation is achieved online processing the images in real time. The use of the Vision Development Module (VDM, National Instruments) allows an easy handling of the images and their subsequent processing.

\section{Sensitivity}

One advantage of LIT is the averaging nature of the method allowing the detection of very small temperature gradients buried in a noisy background. For a LIT experiment, the theoretical performance of the setup is given by the sensitivity $S$ representing the minimal temperature difference that can be measured. It is defined as 


$$
S=\frac{\text { NETD }}{\sqrt{f_{c a m} t_{a c q}}}
$$

where NETD is the Noise Equivalent Temperature Difference or the minimal temperature difference that the infrared camera can resolve in a single image. The desired sensitivity can be obtained by increasing the measurement time or choosing a camera with higher frame rate or lower NETD. The setups presented in Figure 2 are based on a quantum infrared detector exhibiting a NETD of $17 \mathrm{mK}$. An alternative setup, including a standard microbolometer camera, for example the PI-460 (Optris) based on $288 \times 382$ pixels bolometer array demonstrates a NETD of $40 \mathrm{mK}$ for a full frame rate of $80 \mathrm{~Hz}$. As a result, based on Equation 5, we have to measure 17.3 times longer using the microbolometer camera-based setup to achieve the same sensitivity as with the setup using the InSb sensor.

\section{Diffusion length}

When the sample is subjected to a periodic thermal stimulation, highly damped thermal waves will propagate into the medium in all directions on a distance referred to as the thermal diffusion length [15] $\Lambda$, which depends on the thermophysical properties of the medium and on

the pulsation $\Lambda=\sqrt{\frac{2 \alpha}{\omega_{0}}}$, where $\alpha$ is the medium thermal diffusivity. As a result, if the thermal stimulation frequency $\omega_{0}$ is chosen high enough, LIT has the ability to prevent lateral (and longitudinal) heat spreading from the infrared images, resulting in sharp images, which can be particularly interesting when using high magnification objective lenses or to determine the spatial location of NPs in a solid sample. The operating range of the camera in the midwavelength infrared (MWIR, 3-8 $\mu \mathrm{m}$ ) or long-wavelength infrared (LWIR, 8-15 $\mu \mathrm{m}$ ) range drastically limits the maximal resolution achievable using high magnification objectives.

\section{Initial heating}

Digital lock-in demodulation exhibits best results when the measurement is performed under quasi-steady state conditions. This means, the sample temperature oscillates with time around a steady mean temperature. Usually, a thermal relaxation time depending mainly on the heat transfer resistance between the sample surface and the surrounding will take place at the beginning of the experiment (See Part B of Figure 1). This initial period will induce an amplitude and phase shift affecting accuracy of the results. In principle, the quasi-steady state should be reached before demodulating the thermal signal. Nonetheless, such an approach is 
not very practical as depending on the modulation parameters, the initial period can last several minutes. Different methods have been proposed in the literature to solve this problem. In particular, the 'gedankenexperiment' introduced by Breitenstein [15], where the in-phase and in-quadrature images are corrected by subtracting the demodulation of the non-steady period possesses many advantages. The compensated correction takes the form

$$
\begin{gathered}
\operatorname{Corr} 0^{\circ}(x, y)=\frac{\Delta T(x, y)}{N n^{2}} \sum_{j=1}^{n} 2 \mathrm{j} \sin \left(\frac{2 \pi(j-1)}{n}\right) \\
\operatorname{Corr} 90^{\circ}(x, y)=\frac{\Delta T(x, y)}{N n^{2}} \sum_{j=1}^{n} 2 \mathrm{j} \cos \left(\frac{2 \pi(j-1)}{n}\right)
\end{gathered}
$$

where $\Delta T(x, y)$ is the total temperature drift image, and

$$
\begin{gathered}
S_{\text {corr }}^{0^{\circ}}(x, y)=S^{0^{\circ}}(x, y)-\operatorname{Corr} 0^{\circ}(x, y) \\
S_{\text {corr }}^{90^{\circ}}(x, y)=S^{90^{\circ}}(x, y)-\operatorname{Corr} 90^{\circ}(x, y)
\end{gathered}
$$

Allowing the correct calculation of the amplitude and phase.

$$
\begin{gathered}
A(x, y)=\frac{2}{N} \sqrt{S_{\text {corr }}^{0^{\circ}}(x, y)^{2}+S_{\text {corr }}^{0^{\circ}}(x, y)^{2}} \\
\phi(x, y)=\tan ^{-1}\left(\frac{S^{90^{\circ}}(x, y)_{\text {corr }}}{S^{0^{\circ}}(x, y)_{\text {corr }}}\right)
\end{gathered}
$$

We proposed an even simpler compensation method where the initial non-steady-state temperature signal is divided into several segments that are subtracted from the transient temperature. With the digital lock-in demodulation principle being a linear operation, this subtraction can be achieved after demodulation. The amplitude image takes the form after correction

$$
A(x, y)=\sqrt{S^{90^{\circ}}(x, y)^{2}+\left(S^{0^{\circ}}(x, y)-\frac{T_{f-} T_{i}}{n \pi}\right)^{2}}
$$


Similarly to the method proposed by Gupta and Breitenstein, this correction can be performed on-line avoiding the storage of all images onto the computer memory. Nonetheless, it demonstrates lower performances than the "gedankenexperiment".

\section{Non-harmonic heating}

The lock-in demodulation is based on the expectation of a harmonic stimulation. To avoid abrupt variations of the stimulation scheme, the initial phase of the sine or cosine stimulation

signal is set to $\frac{\pi}{4}$. If a non-harmonic stimulation is chosen (like square-wave), the transient thermal signal will contain additional harmonics that will be filtered out if the camera frame rate is much higher that the stimulation frequency. In our different experimental setups where the stimulation frequency usually ranges from 0.1 to $0.8 \mathrm{~Hz}$ and where the camera frame rate is above $80 \mathrm{~Hz}$, we did not notice any significant differences between harmonic and squarewave stimulation. Nonetheless, the shape of the stimulation signal plays a major role when trying to mathematically model the experimental setup to extract quantitative values.

\section{Emissivity compensation}

The emissivity is a unitless quantity describing the ability of the surface of a sample to emit thermal radiation. The emissivity is wavelength dependent and angle dependent. It is usually given for a specific wavelength band (for example LWIR 8-15 $\mu \mathrm{m}$ ) and the angle dependencies are neglected. In LIT, the emissivity will affect identically the in-phase and in-quadrature signal. As a result, the phase remains unaffected by emissivity variations whereas it directly influences the amplitude. When measuring NPs in low concentration, the surface emissivity will be dominated by the medium in which the NPs are embedded. For example, the emissivity of SPIONs diluted in water at different concentrations will remain the surface emissivity of water at ambient temperature (0.96). A way to consider emissivity variations is to introduce a reference with a known emissivity $\varepsilon_{r e f}$ and measure the absolute initial temperature $T_{i}$ of the sample. The radiated power $P_{\text {ref }}$ in Watt of the reference sample is calculated with

$$
P_{\text {ref }}=\varepsilon_{\text {ref }} \sigma \frac{\pi d^{2}}{4}\left(T_{i}^{4}-T_{a}^{4}\right)
$$

where $T_{a}$ the ambient temperature and $\sigma$ the Stephan-Boltzmann constant. The emissivity $\varepsilon$ of an unknown sample can be calculated using 


$$
\varepsilon=\varepsilon_{r e f} \frac{T_{m e a s}^{4}-T_{a}^{4}}{T_{i}^{4}-T_{a}^{4}}
$$

and assigned for each region of interest in the infrared camera software allowing computation of the absolute temperature.

\section{Non-homogeneous stimulation}

When performing LIT experiments, the excitation scheme should be as homogeneous as possible to ensure accuracy of the results. The setup we developed to investigate magnetic NPs (like SPIONs) is based on a commercial AMF generator. AMFs are homogeneous over small spatial regions and as a result, the amplitude will depend on the sample position. On the contrary the phase remains unaffected as the ratio of the in-quadrature and in-phase signals. In previous studies we demonstrated that spatial variations can account for up to $18 \%$ in signal variations [9-14].

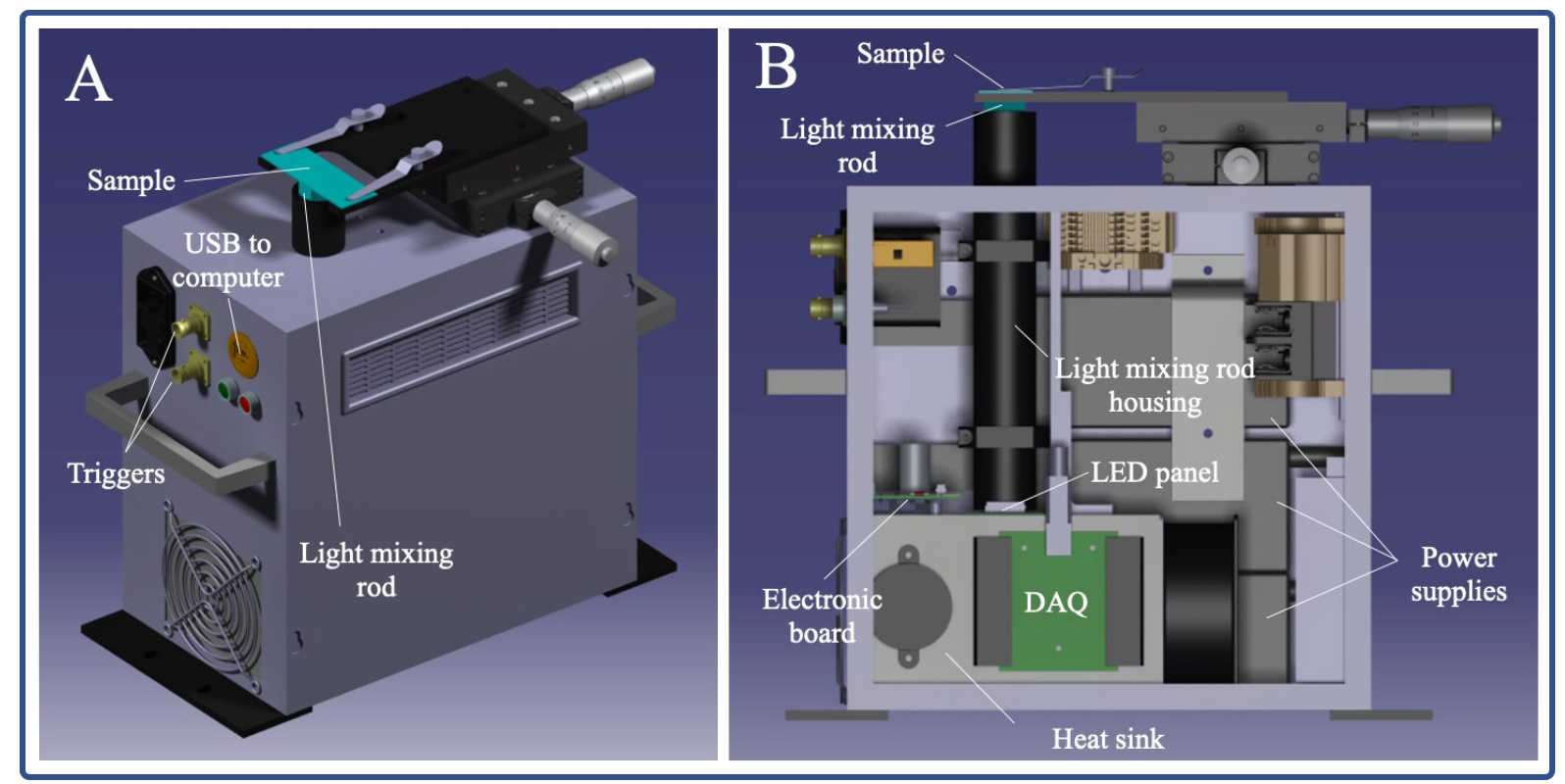

Figure 3: Multiwavelength LED-based light source developed for laboratory use. A: Overview of the custom-made setup. The sample can be positioned with micrometric screws. Trigger input/output lines are connected to the infrared camera, whereas USB connection to the computer enables the selection of wavelength and intensity. B: A custom-made electronic driver is connected to an octagonal-shaped LED panel. To ensure homogeneous illumination, a light mixing rod is inserted between the LEDs and the sample. The LED driver is alimented by 3 power supplies and connected to a DAQ for communication with the computer. To avoid overheating, the LED panel is directly mounted on a heat sink using heat conductive paste. 
To investigate photothermal NPs, we developed a light source consisting in a custom-made octagonal-shaped LED panels emitting stimulation light at various wavelengths (ADOM $\mathrm{GmbH}$ ) (Part B of Figure 2 and Parts A and B of Figure 3). The panel can be designed with a single wavelength for high intensity up to $1 \mathrm{~W} / \mathrm{cm}^{2}$ or incorporate different wavelengths: 420 $\mathrm{nm}, 525 \mathrm{~nm}, 660 \mathrm{~nm}, 730 \mathrm{~nm}, 855 \mathrm{~nm}$ and $940 \mathrm{~nm}$. To ensure homogeneous illumination at the different wavelengths, a light mixing rod (N-BK7, Edmond Optics) is positioned between the sample and the LED array. A custom electronic driver board has been designed to modulate the LED intensities at the stimulation frequency. This board is connected to a computer via a DAQ card (USB 6008, National Instruments) where a simple software (LabVIEW, National Instruments) allows setting the LED intensity as well as the wavelengths to be used. LED performances are dependent on the temperature and in particular the peak wavelength is shifting until the temperature stabilizes. Therefore, the LED panel is mounted on a heat sink connected to a ventilator. A temperature sensor is mounted directly on the LED board to monitor the temperature during use.

\section{Dual Wavelength mode (DWLIT) for measuring plasmonic NPs in strong absorbing medium}

Lock-in detection is a well know approach in engineering that demonstrates the ability to suppress constant slowly varying background signals and to enhance the signal-to-noise-ratio (SNR). Nonetheless, the technique cannot cancel out artefact signals at the stimulation frequency (or lock-in frequency). In the case of photothermal NPs, this issue can arise if the particles are embedded into a strong absorbing medium that is generating heat at the same wavelength as the NPs themselves (see Part A of Figure 4). Following previous solutions developed for similar problems in the field of photovoltaics, we propose a dual wavelength approach (DWLIT) where 2 separate wavelengths are used as stimulation. Both wavelengths are amplitude-modulated at the stimulation frequency but out-of-phase (see part $\mathrm{C}$ of Figure $4)$. 


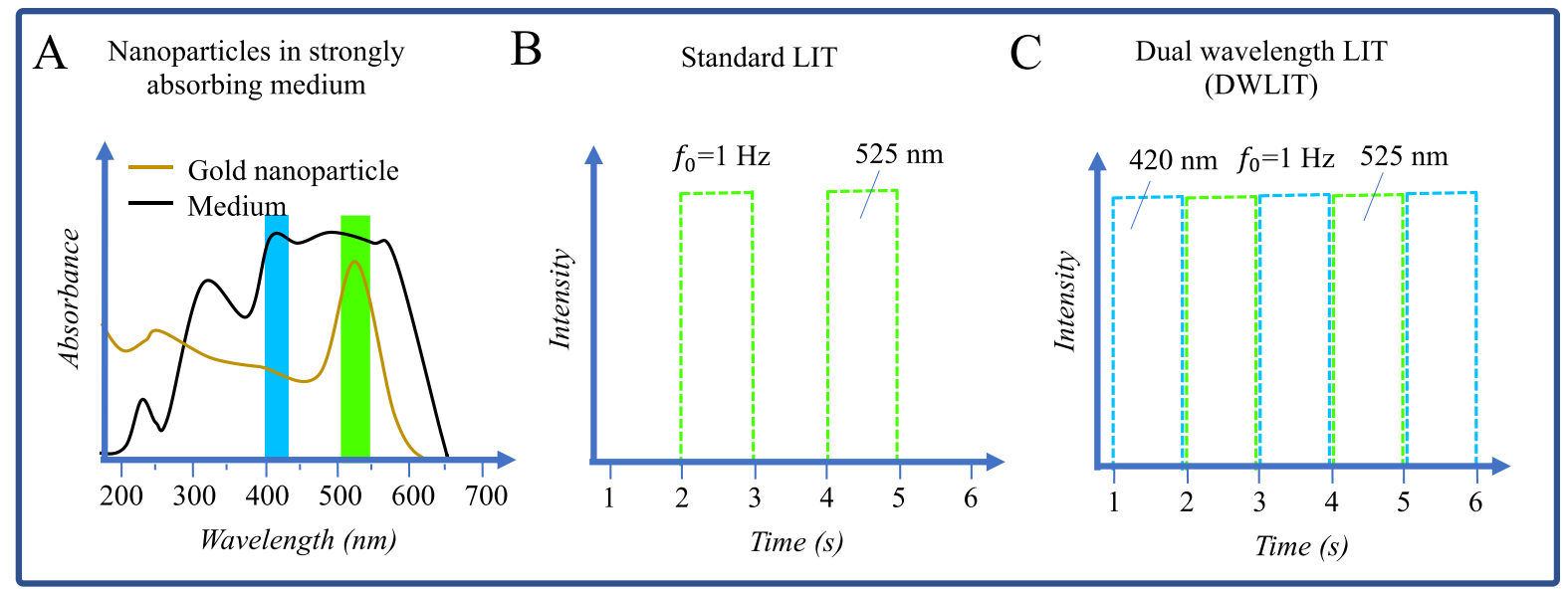

Figure 4: Dual wavelength mode lock-in thermal imaging (DWLIT). A: Pictorial absorption spectrum of spherical gold NPs embedded in an absorbing medium. The NPs exhibit plasmonic-based enhanced absorption at $525 \mathrm{~nm}$ where the medium is also strongly absorbing. Performing standard amplitude-modulated LIT (see part B) illuminating the sample at around $525 \mathrm{~nm}$ would lead to the addition of the heat generated by the medium and the NPs. Performing DWLIT using an additional wavelength (420 nm for example) where the medium absorption is similar to $525 \mathrm{~nm}$ allows the lock-in principle to reject the heat generated by the medium (see part C). B: Standard LIT where the stimulation signal amplitude is modulated at a defined stimulation frequency ( $1 \mathrm{~Hz}$ in this example). C: DWLIT where the stimulation wavelength is switched between $420 \mathrm{~nm}$ and $525 \mathrm{~nm}$ allowing a better rejection of the heat generated by the medium.

\section{Advantages of LIT for the investigation of NPs}

In recent review articles, common analytical methods are evaluated in detail and classified with respect to their applicability and flexibility for NP characterization. They generally share the opinion that (i) the current measures for the detection and quantification of NPs in complex matrices are not yet sufficiently developed, (ii) depending on the given matrix potential interference signals and/or artifacts have to be reliably filtered, (iii) the majority of the currently available tools are useful but often very time-consuming, complex and demand great expertise in terms of operation and data treatment, (iv) no single method is capable of resolving all relevant material-related parameters and NP types, and that (v) novel, affordable and straightforward analytical methods should be developed. In general, main analytical challenges are related to several distinct parameters including e.g. the low concentration of NPs in products, the complexity and variability of the matrix (including solids, gels, cells etc.), the presence of potentially high concentrations of proteins, electrolytes, synthetic polymers, binder, or pigments, or the change of NP composition (e.g. dissolution), dispersion state (i.e. agglomeration), or surface chemistry. 
Existing techniques can be very powerful for specific NPs embedded or suspended in particular matrices/liquids, but frequently face detrimental limitations when the environmental conditions are altered. As a consequence, plenty of complementary analytical methods have emerged over the last decades, all with certain advantages, but also constraints. Especially heterogenous and polydisperse samples have proven to be consistently demanding when it comes to their analysis. Many techniques are highly complex in terms of sample preparation, operation, and data analysis, rendering them time- and cost-intensive. A common fact is that they are also rarely available and therefore restricted in access. Table 1 presents the different standard methods currently used to investigate NPs in complex environments depending on the objectives.

\begin{tabular}{|c|c|c|c|}
\hline Objectives & Current method of choice & Limitations & $\begin{array}{c}\text { Advantages of Lock-in Thermal } \\
\text { Imaging }\end{array}$ \\
\hline $\begin{array}{l}\text { Quantification of nanoparticles in a solid } \\
\text { matrix }\end{array}$ & Inductively Coupled Plasma (ICP) & $\begin{array}{l}\text { - Destructive } \\
\text { Differentiation between metal } \\
\text { nanoparticles and } \mathrm{Me}^{\mathrm{x}+}-\mathrm{Ion} \\
\text { impossible }\end{array}$ & $\begin{array}{l}\text { - In-situ technique, non-destructive } \\
\text { - } \text { Specific for nanoparticles } \\
\text { 2D spatial information }\end{array}$ \\
\hline $\begin{array}{l}\text { Detection and characterization of } \\
\text { nanoparticles in suspension }\end{array}$ & Dynamic Light Scattering (DLS) & $\begin{array}{l}\text { - Limited to samples in liquid } \\
\text { Non-purified and } \\
\text { heterogeneous samples are } \\
\text { problematic }\end{array}$ & $\begin{array}{l}\text { - 2D spatial information } \\
\text { - Liquid/solid can be investigated }\end{array}$ \\
\hline \multirow{3}{*}{$\begin{array}{l}\text { Detection and characterization of } \\
\text { nanoparticles in biological environments }\end{array}$} & $\begin{array}{l}\text { Transmission Electron Microscopy } \\
\text { (TEM) \& Scanning Electron } \\
\text { Microscopy (SEM) }\end{array}$ & $\begin{array}{l}\text { - } \text { Time intensive } \\
\text { - } \\
\text { Sxpensive } \\
\text { and/or sectioned }\end{array}$ & \multirow{3}{*}{ 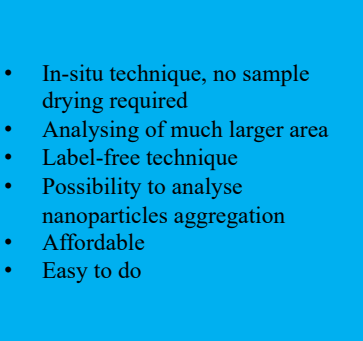 } \\
\hline & Fluorescent Microscopy (FM) & $\begin{array}{l}\text { Fluorescent labelling necessary } \\
\text { (nanoparticles surface } \\
\text { modification) }\end{array}$ & \\
\hline & $\begin{array}{l}\text { Dark-field Microscopy } \\
\text { (DM) }\end{array}$ & $\begin{array}{l}\text { Expensive } \\
\text { Expert knowledge for data } \\
\text { acquisition and analysis } \\
\text { required }\end{array}$ & \\
\hline $\begin{array}{l}\text { Quantification of carbon-based } \\
\text { nanoparticles in a matrix }\end{array}$ & No methods currently available & No methods currently available & $\begin{array}{l}\text { Carbon-containing nanoparticles } \\
\text { can be detected and ultimately } \\
\text { quantified }\end{array}$ \\
\hline
\end{tabular}

The extraction of NPs from their solid matrix is often inevitable to perform proper NP investigations (e.g. Inductively Coupled Plasma - ICP). Nonetheless, such measurements are destructive and besides, the differentiation with charged NPs can be cumbersome. LIT offers an in-situ alternative that is nondestructive and NP specific.

To investigate NPs in suspension, dynamic light scattering (DLS) is arguably the most common method offering high sensitivity and resolving even small NPs. However, measuring heterogenous and non-purified samples becomes extremely challenging. In contrary, LIT can measure both samples in liquid and solid states and thanks to the imaging nature of the techniques, multiple samples can be investigated simultaneously in a time efficient manner. In biological media, investigations are often achieved using imaging methods based on Electron Microscopy (EM), Fluorescence Microscopy (FM) or Dark-field Microscopy (DM). For high resolution NP visualization, EM is the number one option, but is usually fairly time-intensive, 
expensive and the obtained images are not necessarily representative for the entire sample. In biological settings, FM is often applied to evaluate NP uptake. Nonetheless, the technique requires dye-labelling of NPs and recent studies have demonstrated that the presence of dyes can significantly alter NP-cell interactions, leading to potential misinterpretation of the data. On the other hand, DM is an expensive method and requires expertise for the interpretation of the data. Even if LIT cannot provide similar spatial resolution as EM or FM, it allows to gain an overview of the overall sample, does not required any sample preparation or labelling and is cost effective. Finally, a reliable method is lacking for the investigation of carbon-containing NPs due to their complexity. Preliminary investigations with LIT are very promising and highlight that LIT can be used for the precise estimation of carbon nanotube content in biological environments in a time-efficient and label-free fashion.

\section{Summary}

The increase use of NPs in a large variety of products triggers the necessity to develop analytical tools that can detect and characterize NPs in complex but rather common environments like cell cultures or gels. Among the current methods available, LIT is a promising technique, particularly suited for stimuli-responsive NPs that generate heat upon a triggerable stimulation like light or an AMF. In this work we presented the core features of lock-in thermal imaging describing in detail the setups, algorithms and key parameters. We also proposed a further potential improvement in the case of highly absorbing surrounding medium. Finally, we compared lock-in thermal imaging with standard methods demonstrating that it possesses the required sensitivity and versatility to become essential in the field.

\section{Conflict of interest}

Mathias Bonmarin and Christoph Geers are the co-founders of NanoLockin $\mathrm{GmbH}$, a company developing and commercializing lock-in thermal imaging apparatus to measure stimuliresponsive NPs.

\section{References}

[1] S. Moeinzadeh, and E. Jabbari, "Nanoparticles and Their Applications," Springer Handbook of Nanotechnology, B. Bhushan, ed., pp. 335-361, Berlin, Heidelberg: Springer Berlin Heidelberg, 2017.

[2] J. Zhang, L. Chen, W. H. Tse, and R. Bi, "Inorganic Nanoparticles: Engineering for Biomedical Applications," IEEE Nanotechnology Magazine, vol. 8, no. 4, pp. 21-28, 2014.

[3] E. K. Richman, and J. E. Hutchison, "The Nanomaterial Characterization Bottleneck," Acs Nano, vol. 3, no. 9, pp. 2441-2446, Sep, 2009. 
[4] G. Busse, D. Wu, and W. Karpen, "Thermal wave imaging with phase sensitive modulated thermography," Journal of Applied Physics, vol. 71, no. 8, pp. 3962-3965, 1992.

[5] M. Vollmer, and K.-P. Möllman, Infrared Thermal Imaging: Fundamentals, Research and Applications: Wiley-VCH, 2010.

[6] K. M. Donnell, "Active microwave thermography - a new twist on microwave NDT [In Microwave Women Research]," IEEE Instrumentation \& Measurement Magazine, vol. 19, no. 3, pp. 24-26, 2016.

[7] A. Mirala, A. Foudazi, M. T. A. Qaseer, and K. M. Donnell, "Active Microwave Thermography to Detect and Locate Water Ingress," IEEE Transactions on Instrumentation and Measurement, pp. 1-1, 2020.

[8] B. Gao, L. Bai, W. L. Woo, G. Y. Tian, and Y. Cheng, "Automatic Defect Identification of Eddy Current Pulsed Thermography Using Single Channel Blind Source Separation," IEEE Transactions on Instrumentation and Measurement, vol. 63, no. 4, pp. 913-922, 2014.

[9] L. Steinmetz, J. Bourquin, H. Barosova, L. Haeni, J. Caldwell, A. Milosevic, C. Geers, M. Bonmarin, P. Taladriz-Blanco, B. Rothen-Rutishauser, and A. Petri-Fink, "Rapid and sensitive quantification of cell-associated multi-walled carbon nanotubes," Nanoscale, vol. 12, no. 33, pp. 17362-17372, 2020.

[10] L. Steinmetz, C. Kirsch, C. Geers, A. Petri-Fink, and M. Bonmarin, "Investigating a Lock-In Thermal Imaging Setup for the Detection and Characterization of Magnetic Nanoparticles," Nanomaterials (Basel, Switzerland), vol. 10, no. 9, 2020 Aug, 2020.

[11] L. Steinmetz, P. Taladriz-Blanco, C. Geers, M. Spuch-Calvar, M. Bonmarin, S. Balog, B. Rothen-Rutishauser, and A. Petri-Fink, "Lock-In Thermography to Analyze Plasmonic Nanoparticle Dispersions," Particle \& Particle Systems Characterization, vol. 36, no. 9, Sep, 2019.

[12] P. Lemal, C. Geers, C. A. Monnier, F. Crippa, L. Daum, D. A. Urban, B. RothenRutishauser, M. Bonmarin, A. Petri-Fink, and T. L. Moore, "Lock-in thermography as a rapid and reproducible thermal characterization method for magnetic nanoparticles," Journal of Magnetism and Magnetic Materials, vol. 427, pp. 206-211, Apr, 2017.

[13] C. A. Monnier, F. Crippa, C. Geers, E. Knapp, B. Rothen-Rutishauser, M. Bonmarin, M. Lattuada, and A. Petri-Fink, "Lock-In Thermography as an Analytical Tool for Magnetic Nanoparticles: Measuring Heating Power and Magnetic Fields," Journal of Physical Chemistry C, vol. 121, no. 48, pp. 27164-27175, Dec, 2017.

[14] C. A. Monnier, M. Lattuada, D. Burnand, F. Crippa, J. C. Martinez-Garcia, A. M. Hirt, B. Rothen-Rutishauser, M. Bonmarin, and A. Petri-Fink, "A lock-in-based method to examine the thermal signatures of magnetic nanoparticles in the liquid, solid and aggregated states," Nanoscale, vol. 8, no. 27, pp. 13321-13332, 2016.

[15] O. Breitenstein, W. Warta, and M. Langenkamp, Lock-in Thermography: Basics and Use for Evaluating Electronic Devices and Materials: Springer, 2010. 


\section{Biographies}

Mathias Bonmarin (mathias.bonmarin@zhaw.ch) is currently Professor at the Zurich University of Applied Sciences, School of Engineering where he is leading the Sensors and Measurement Systems group. Mathias studied at the graduate school of science and engineering "POLYTECH Marseille" part of Aix-Marseille University and obtained his master's degree in biomedical engineering in 2002. He also owns an additional master in optics, optoelectronics and microwave from the Grenoble Institute of Technology and a master in economics from the University of Louvain-La-Neuve in Belgium. Mathias got his $\mathrm{PhD}$ in physical chemistry at the University of Zurich in 2010. His research interests include the development of innovative sensors and methods for applications in the medicine and biology.

Lukas Steinmetz (lukas.steinmetz@unifr.ch) is currently employed as a postdoctoral researcher at the Chemistry Department of the University of Fribourg. He obtained his master's degree in 'Geomaterials and Geochemistry' from the LMU \& TU Munich in 2016. Subsequently, he joined the Bionanomaterials group of the Adolphe Merkle Institute in Fribourg as a doctoral student, where he defended his $\mathrm{PhD}$ in materials science in summer 2020. His research is focused on the analysis of metallic nanomaterials and their detection in complex environments.

Fabrizio Spano (fabrizio.spano@zhaw.ch) is currently employed as senior research associate at the Zurich University of Applied Sciences, School of Engineering, at the Institute of Computational Physics in the Sensors and Measurement Systems group. Fabrizio got his PhD in Chemistry at the University of Haute-Alsace (Mulhouse, France) in 2003. His research interests are oriented towards material science, including the elaboration of composite and nanocomposites for various applications and in particular towards the design and development of artificial skin models. 